\title{
Incidence and risk factors of dysphagia after variceal band ligation
}

\author{
Saraswathi Arasu', Hammad Liaquat', Jaspreet Suri², Adam C. Ehrlich', and Frank K. Friedenberg' \\ 'Gastroenterology Section and ${ }^{2}$ Internal Medicine, Department of Medicine, Temple University School of Medicine, Philadelphia, PA, \\ USA
}

Background/Aims: There is a lack of data on long-term morbidity, particularly dysphagia, following endoscopic variceal band ligation (EVL). The aim of this study are to assess the incidence of dysphagia and variables associated with this complication after EVL.

Methods: We identified individuals who completed at least one session of EVL as their sole treatment for varices from August 2012 to December 2017. Included patients achieved "complete eradication" of varices not requiring further therapy. Patients $\geq 90$ days from their last EVL session completed a modified version of the Mayo Clinic Dysphagia Questionnaire. Individuals with dysphagia were invited to undergo a barium esophagram. Patients with pre-EVL dysphagia were excluded.

Results: Of the patients, 68 possessed inclusion criteria, nine (13.2\%) died and 20 (29.4\%) were lost to follow up. For the remaining 39 (57.4\%) patients, 23 were males, mean age of $61.7 \pm 8.6$ years. The most common etiology of liver disease was hepatitis $C$ virus $(n=18 ; 46.2 \%)$. The median number of banding sessions was 2.0 (interquartile range [IQR], 1.0-4.0) with a median of 9.0 bands placed (IQR, 3.0-14.0). Twelve patients (30.8\%) developed new-onset dysphagia post-EVL. In univariate analysis, pre-EVL MELD score and non-emergent initial banding were associated with long-term dysphagia. In a regression model adjusted for age, sex, number of bands, and use of acid suppression after EVL, no factor was independently associated with dysphagia (all $P>0.05$ ). No strictures were identified on subsequent esophageal evaluation.

Conclusions: Approximately $30 \%$ of patients developed new-onset, chronic dysphagia post-EVL. Incident dysphagia was associated with a non-emergent initial banding session. The mechanism for dysphagia remains unknown. (Clin Mol Hepatol 2019;25:374-380)

Keywords: Esophageal and gastric varices; Deglutition disorders; Esophageal stenosis

\footnotetext{
Study Highlights

This investigation evaluated the prevalence of dysphagia post-endoscopic variceal band ligation (EVL) and identified variables that may be associated with dysphagia. Our study showed that nearly $1 / 3$ of patients developed new onset dysphagia post-EVL; however, there were no variables independently associated with this outcome in logistic regression analysis. This manuscript will be of interest to not only clinical investigators in the area of the esophagus, but also to physicians caring for the many patients who have decompensated cirrhosis requiring esophageal variceal band ligation.
}

\section{Abbreviations:}

$\mathrm{Cl}$, confidence interval; EVL, endoscopic variceal band ligation; EVS, endoscopic variceal sclerotherapy; $H C V$, hepatitis $C$ virus; IQR, interquartile range; $M D Q$, Mayo Dysphagia Questionnaire; MELD, model for end-stage liver disease; OR, odds ratio

\author{
Corresponding author : Frank K. Friedenberg \\ Gastroenterology Section, Department of Medicine, Temple University \\ School of Medicine, 3401 North Broad Street, Philadelphia, PA 19140, USA \\ Tel: +1-215-707-7579, Fax: +1-215-707-2684 \\ E-mail: frank.friedenberg@tuhs.temple.edu \\ https://orcid.org/0000-0003-1733-320X
}




\section{INTRODUCTION}

Bleeding esophageal varices are the most severe complication of portal hypertension and represent the leading cause of death in patients with cirrhosis. Esophageal varices are present in approximately $50 \%$ of patients with cirrhosis. ${ }^{1}$ In the past, endoscopic variceal sclerotherapy (EVS) was the intervention of choice in bleeding esophageal varices, but currently endoscopic variceal band ligation (EVL) is thought to be a safer procedure with fewer complications. $^{2,3}$ In the immediate setting, EVS can result in bacteremia, which can lead to metastatic infections including pneumonia and bacterial peritonitis. ${ }^{4}$ Long-term data has demonstrated that after EVS esophageal stricture formation is relatively common. ${ }^{5}$ Additionally, EVS has been associated with esophageal motor disorders including a decrease in esophageal peristaltic wave amplitude and an increase in simultaneous contractions. ${ }^{6,7}$

Complications of EVL occur in approximately $14 \%$ of cases but are usually minor. ${ }^{8,9}$ For example, bacteremia is not seen with EVL. The most common complications are transient dysphagia and chest discomfort. ${ }^{8,9}$ These are related to shallow ulcers which develop post-EVL but heal within 4 weeks of treatment. ${ }^{10}$ Surprisingly, there is limited long-term data evaluating esophageal symptoms as well as structural and functional abnormalities after EVL. Therefore, the objectives of the study were to: 1) assess the incidence of long-term (>90 days) dysphagia in patients after EVL; 2) identify clinical factors associated with dysphagia; and 3) assess the incidence of esophageal structural disorders after EVL.

\section{MATERIALS AND METHODS}

\section{Study population}

We identified all patients who underwent upper endoscopy for emergent or elective evaluation and treatment of esophageal varices between August 2012 and December 2017 at our institution. Subjects were identified via the electronic medical record using Provation (ProVation Medical, Minneapolis, MN, USA). Our objective was to identify patients who underwent one or more EVL sessions and at the conclusion were felt to no longer need EVL. Therefore, to be eligible for study inclusion, patients needed to meet all of the following criteria: 1) documented EVL for the treatment of esophageal varices due to portal hypertension; 2) documentation that no further EVL sessions were needed and that only small residual varices were present; and 3) patients must have completed $E V L \geq 90$ days prior to enrollment. We chose a minimum of 90 days to allow for healing of acute ulceration and to allow for the development of fibrotic changes to the mucosa and/or deeper layers of the esophagus post-EVL. We defined dysphagia present $\geq 90$ days post-EVL as long-term dysphagia. Patients were excluded from the study if they met any of the following criteria: 1) a history of dysphagia prior to $E V L ; 2$ ) a history of any disorder associated with esophageal dysphagia prior to banding such as Scleroderma and peptic esophagitis/stricture; 3) use of sclerosant during the ablation of varices; and 4) prior gastric or esophageal surgery. Our policy is to perform EVL every 2-4 weeks until varices are obliterated however following this protocol was not a determinant of study inclusion.

\section{Study design}

The design was a single-center study. Patients were identified retrospectively but interviewed prospectively. Patients meeting the study's inclusion/exclusion criteria were interviewed in the office. The study protocol was described and informed consent was obtained which was approved by Institutional Review Board (IRB) of Temple University School of Medicine (IRB No. 23996). Eligible patients were administered a modified version of the Mayo Dysphagia Questionnaire (MDQ)." The full MDQ contains 28 items however we retained only those questions relevant to our study. The retained eight questions focus solely on dysphagia (Fig. 1). If question 1, "Do you have trouble swallowing solid food?" was answered with "yes", the patient was considered to have dysphagia. The remaining questions quantify the frequency of dysphagia and types of foods the patient finds troublesome to swallow. From the questionnaire, patients identified as having dysphagia were invited to undergo a barium esophagram which included ingestion of a $13 \mathrm{~mm}$ barium-impregnated pill.

Demographic data such as age, sex, and etiology of liver disease were recorded. We recorded the model for end-stage liver disease (MELD) score and Child-Turcotte-Pugh class for patients both pre- and post-EVL. Endoscopic variables collected included the presence of a hiatal hernia, whether the original bands were placed electively or emergently, number of banding sessions, total number of bands placed, and whether the patient was placed on a proton pump inhibitor after the procedure. We reviewed each study to determine whether an esophageal stricture was noted during or after banding. 


\section{Statistical analysis}

We performed a univariate analysis comparing variables between subjects who did and did not develop dysphagia after banding. Comparison of continuous data was performed using Student's $t$-test after confirming a normal distribution of data points. For categorical variables, a chi-square or Fisher Exact test was used. For non-parametric data, appropriate formulas such as Mann-Whitney $U$ or Kruskall-Wallis were used. All analyses were 2-tailed with a significance level (a) set at 0.05 .

All significant $(P \leq 0.05)$ predictors of dysphagia from univariate analysis were entered into a logistic regression model. Using dysphagia as the outcome (dependent variable), a stepwise forward entry of predictor (independent) variables was performed to determine if any variables were independently associated with dysphagia. Data output was expressed as the adjusted odds ratio with corresponding 95\% confidence interval (CI). SPSS Statistics ver. 24 (IBM, Armonk, NY, USA) was used for statistical analysis.

\section{RESULTS}

During the period of August 2012 and December 2017, 68 patients met study inclusion/exclusion criteria. However, nine (13.2\%) expired and 20 (29.4\%) were lost to follow-up (Fig. 2). For the evaluable $39(57.4 \%)$ patients, there were $23(59 \%)$ males and $16(41 \%)$ females with a mean age of $61.7 \pm 8.6$ years (Table 1). The major etiologies of liver disease included hepatitis $C$ virus (HCV) $(n=18 ; 46.2 \%)$, non-alcoholic steatohepatitis $(n=7 ; 17.9 \%)$, and alcoholic liver disease $(n=7 ; 17.9 \%)$. For those with HCV a similar proportion had viral eradication prior to EVL. The median number of banding sessions, including the index session if performed for bleeding, was 2.0 (interquartile range [IQR], 1.0-4.0), with a range of $1-5$ sessions. The median total number of bands placed per patient was 9.0 (IQR, 3.0-14.0), with a range of 1 to 30 bands.

Twelve patients (30.8\%) developed new onset dysphagia postEVL. The table compares the characteristics of patients who did and did not develop dysphagia. For those with dysphagia, with

Please focus on your symptoms over the past 30 days.

1. Do you have trouble swallowing solid food?

(0) No

(1) Yes

2. In the past $\mathbf{3 0}$ days, has your swallowing trouble gotten better, remain unchanged, or gotten worse?

(1) Better

(2) Unchanged

(3) Worse

3. How often have you had difficulty swallowing solid food in the past 30 days?

(1) Less than once a week

(2) Once a week

(3) Several times a week

(4) Daily

(5) With every meal

(6) With each swallow

(7) Unable to eat

\author{
4. In the past $\mathbf{3 0}$ days, have you avoided any of the following types of \\ foods to avoid food getting stuck? \\ (Mark "Yes" or "No" for all) \\ Oatmeal (other foods, like grits, cream of wheat, rice) \\ (1) $\mathrm{Y} \quad(0) \mathrm{N}$ \\ Banana (or other foods, like pudding, jello) \\ (1) $\mathrm{Y} \quad(0) \mathrm{N}$ \\ Apple (or other foods with fiber, such as celery) \\ (1) Y (0) N \\ Ground meat (like hamburger or ground turkey) \\ (1) $\mathrm{Y} \quad(0) \mathrm{N}$ \\ Bread (other foods, like cake, doughnuts, muffins) \\ $\begin{array}{ll}\text { (1) } \mathrm{Y} & \text { (0) } \mathrm{N}\end{array}$ \\ Meat (fibrous meats, like steak or chicken) \\ (1) $\mathrm{Y}$ (0) $\mathrm{N}$
}

\author{
5. In the past $\mathbf{3 0}$ days, have you had trouble swallowing any of these \\ foods or other foods like them? \\ (Mark "Yes" or "No" for all) \\ Oatmeal (other foods, like grits, cream of wheat, rice) \\ (1) $\mathrm{Y} \quad(0) \mathrm{N}$ \\ Banana (or other foods, like pudding, jello) \\ (1) $\mathrm{Y}(0) \mathrm{N}$ \\ Apple (or other foods with fiber, such as celery) \\ (1) $\mathrm{Y}(0) \mathrm{N}$ \\ Ground meat (like hamburger or ground turkey) \\ (1)Y (0) N \\ Bread (or other foods, like cake, doughnuts, muffins) \\ (1) $\mathrm{Y}(0) \mathrm{N}$ \\ Meat (fibrous meats, like steak or chicken) \\ (1) $\mathrm{Y}(0) \mathrm{N}$
}

\begin{abstract}
6. Do you modify your food (such as boil, use a blender, cut into smal pieces, or dunk in liquid) to make it easier to swallow?

(0) No

(1) Yes

7. Do you have problems swallowing liquids in the last 30 days?

(0) No

(1) Yes
\end{abstract}

8. In the past $\mathbf{3 0}$ days, have you had difficulty swallowing pills? (0) No

(1) Yes

Figure 1. Modified version of the Mayo Dysphagia Questionnaire. The full questionnaire includes 28 questions; we used only 8 that query dysphagia. Adapted from McElhiney et al." with permission from the Mayo Clinic, Rochester MN. 
respect to difficulty swallowing food in the past 30 days, 50\% had difficulty once per week, 25\% less than once per week, and $16.6 \%$ several times per week. To prevent food "getting stuck",

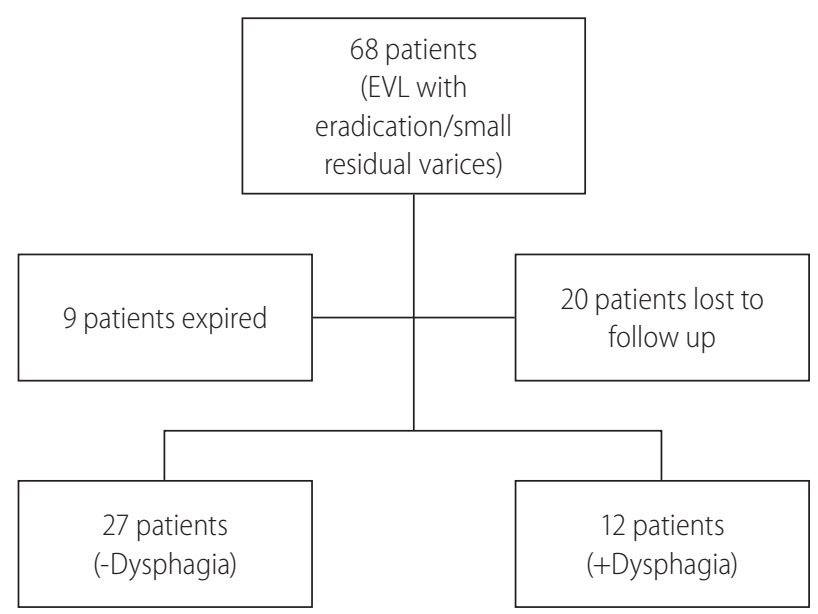

Figure 2. Flow diagram of patient inclusion in the study. Patients with dysphagia answered "Yes" to the question "Do you have trouble swallowing solid food?". EVL, endoscopic variceal band ligation.
58.3\% avoided meat, 25\% avoided apples, and 25\% avoided ground meat, with smaller percentages for other foodstuffs. Asked another way, 66\% had trouble swallowing meat, 33\% had trouble swallowing ground meat, and 25\% had trouble swallowing apples. When asked whether post-EVL dysphagia sufferers modified their diet to make it easier to swallow, 50\% answered "yes", 50\% "no". With respect to liquid dysphagia, 91.7\% responded "no" and 8.3\% "yes". For difficulty swallowing pills, $66 \%$ responded "no" and 33\% "yes".

On univariate analysis, the variables associated with dysphagia post-completion of EVL were the proportion of cases where the initial procedure was performed non-emergently vs. emergently (40.7 vs. $8.3 \%, P=0.043$ ) and the pre-EVL MELD score for those with (10.8 \pm 3.0$)$ and without $(15.0 \pm 6.8)$ dysphagia $(P=0.01)$. We performed a stepwise forward binary logistic regression, controlling for age, gender, total number of bands placed, and whether proton pump inhibitor therapy was used after EVL. After adjustment, both pre-EVL MELD score (adjusted odds ratio [OR], 0.84; 95\% Cl, 0.69-1.02; $P=0.07)$ and whether the first banding session was emergent (adjusted OR, 8.32; 95\% Cl, 0.74-93.6;

Table 1. Comparison of patients with and without dysphagia after banding

\begin{tabular}{|c|c|c|c|}
\hline & No dysphagia $(n=27)$ & Dysphagia $(n=12)$ & $P$-value \\
\hline Male & $17(62.3)$ & $6(50.0)$ & 0.45 \\
\hline Mean age (years) & $60.8 \pm 9.5$ & $63.6 \pm 6.3$ & 0.36 \\
\hline Hemoglobin A1C & $6.5 \pm 2.5$ & $6.9 \pm 2.4$ & 0.71 \\
\hline Etiology of liver disease & & & 0.14 \\
\hline Hepatitis C & $11(40.7)$ & $7(58.3)$ & \\
\hline $\mathrm{NASH}$ & $6(22.2)$ & $1(8.3)$ & \\
\hline Alcoholic & $5(18.5)$ & $2(16.7)$ & \\
\hline Other & $5(18.5)$ & $2(16.7)$ & \\
\hline \multicolumn{4}{|l|}{ MELD score } \\
\hline Prior to banding & $15.0 \pm 6.8$ & $10.8 \pm 3.0$ & 0.01 \\
\hline After banding & $14.1 \pm 8.4$ & $13.3 \pm 6.6$ & 0.74 \\
\hline \multicolumn{4}{|l|}{ Child-Turcotte-Pugh score } \\
\hline Prior to banding & $8.1 \pm 2.1$ & $7.8 \pm 1.9$ & 0.73 \\
\hline After banding & $7.8 \pm 2.4$ & $7.8 \pm 2.1$ & 0.98 \\
\hline Initial EGD emergent & $11 \pm 40.7$ & $1 \pm 8.3$ & 0.04 \\
\hline Median number of EVL sessions & $2.0(1.0-3.0)$ & $2.0(1.0-4.0)$ & 0.63 \\
\hline Median total number of bands & $11.0(6.0-14.0)$ & $5.5(2.0-18.75)$ & 0.21 \\
\hline Presence of hiatal hernia & $5(18.5)$ & $4(33.3)$ & 0.32 \\
\hline Proton Pump Inhibitor (\%) & 74.1 & 58.3 & 0.33 \\
\hline
\end{tabular}

Values are presented as mean \pm standard deviation, number (\%), or number (interquartile range).

NASH, non-alcoholic steatohepatitis; MELD, model for end-stage liver disease; EGD, esophagogastroduodenoscopy; EVL, endoscopic variceal ligation. 
Table 2. Output from multivariate analysis comparing patients with and without dysphagia after banding

\begin{tabular}{lcccc}
\hline & No dysphagia $(\mathbf{n = 2 7})$ & Dysphagia $(\mathbf{n = 1 2})$ & Adjusted OR* (95\% Cl) & $\boldsymbol{P}_{\text {-value }}$ \\
\hline MELD score & & & & \\
$\quad$ Prior to banding & $15.0 \pm 6.8$ & $10.8 \pm 3.0$ & $0.84(0.69-1.02)$ & 0.07 \\
After banding & $14.1 \pm 8.4$ & $13.3 \pm 6.6$ & & \\
Initial EGD emergent (\%) & $11(40.7)$ & $1(8.3)$ & $8.32(0.74-93.6)$ & 0.08
\end{tabular}

Values are presented as mean \pm standard deviation or number (\%) unless otherwise indicated.

$O R$, odds ratio; $\mathrm{Cl}$, confidence interval; $M E L D$, model for end-stage liver disease; $E G D$, esophagogastroduodenoscopy.

*Odds ratio adjusted for age, gender, median number of banding sessions, and use of proton pump inhibitors after banding sessions.

$P=0.08)$ were no longer significantly associated with post-EVL dysphagia (Table 2).

\section{Evaluation for esophageal stricture}

None of the twelve cases of new onset dysphagia post-EVL were identified to have a stricture on their final upper endoscopy as part of EVL treatment. Only five of 12 agreed to undergo an esophagram post-EVL; three were normal, one showed a diffuse abnormality of peristalsis, and one demonstrated a focal mural irregularity without luminal narrowing in the distal esophagus. One patient underwent upper endoscopy for another indication 16 months after completing EVL and no stricture was identified.

\section{DISCUSSION}

The current study investigated the incidence and potential variables associated with dysphagia in patients $\geq 90$ days after complete eradication of all varices with band ligation. At this point in time, we know that there is long-term treatment-related morbidity associated with EVS (approximately 40\%) and short-term morbidity with EVL including transient dysphagia. ${ }^{12,13}$ Our study is a unique investigation because the prevalence of long-term complications from EVL has not been carefully studied.

We explored several relevant potential risk factors for dysphagia post-EVL including age, sex, number of banding sessions, number of bands placed, and whether the index EVL was performed emergently. The median number of EVL sessions in our study to achieve eradication was 2.0, similar to findings in other studies. ${ }^{14}$ We found on univariate analysis that a lower MELD score and whether the index EVL was performed non-emergently for bleeding were predictors of experiencing dysphagia as a long-term morbidity. However, after controlling for relevant potential confounders using logistic regression modeling, neither of these vari- ables was shown to be independently associated with post-EVL dysphagia.

In 1989, Van Stiegmann and Goff ${ }^{9}$ and Van Stiegmann et al. ${ }^{15}$ introduced the application of EVL. In their study they identified that all of their patients achieving complete variceal eradication reported mild dysphagia for 12-24 hours following EVL but none required active treatment or developed strictures. The results of the study highlighted that EVL is a safe alternative to EVS but did not look at long-term complications. Similarly, Viazis et al. ${ }^{4}$, compared the short-term effects of EVL and EVS after variceal eradication on esophageal motility. This randomized study of 60 patients found that none treated with EVL $(n=30)$ complained of reflux symptoms or dysphagia after completion of endoscopic treatment but follow-up was only for 6 weeks.

The technique used for EVL obliterates varices by causing mechanical strangulation with rubber bands, an adaptation of the concept applied to band ligation of internal hemorrhoids. The main reaction is usually limited over the superficial esophageal mucosa where ischemic necrosis occurs, followed by granulation tissue formation and sloughing of the bands. ${ }^{13}$ This may lead to shallow mucosal ulcerations and complete re-epithelialization of the mucosa with maturing scar tissue that can heal over time. ${ }^{16}$ We chose $\geq 90$ days after the patient's final EVL session as this should allow for sufficient time for edema and superficial mucosal ulceration to resolve. Additionally, we assumed early stricture formation would be present by this time if it were going to develop.

In our study we identified that nearly one third of patients developed new-onset dysphagia $\geq 90$ days post-EVL when a modified version of the Mayo Dysphagia Questionnaire was administered. ${ }^{11}$ This is novel information as again, it is common to have transient dysphagia immediately post-banding, however the assumption has been that the dysphagia will resolve over time. While dysphagia prevalence was high, there was no evidence that EVL caused significant structural abnormalities of the esophagus for those that completed a barium esophagram or upper endos- 
Saraswathi Arasu, et al.

Dysphagia after variceal band ligation

copy.

Because stricture formation post-EVL appears to be very rare, the etiology of dysphagia remains elusive. Esophageal motility was not systematically evaluated in this study although one patient was shown to have a diffuse, non-specific peristaltic disorder of the esophageal body post-EVL on barium esophagram. In contrast, data from Viazis et al. ${ }^{4}$, demonstrated that EVL has no impact on esophageal motility unlike EVS, which the authors found leads to a significant decrease of the amplitude of peristaltic waves, an increase of simultaneous contractions in the tubular esophagus, and an increase in pathological gastroesophageal reflux disease. Similarly, a case series published by Berner et al. ${ }^{17}$, demonstrated that the majority of EVL treatments did not result in a worsening of motility or reflux scores and none were associated with symptoms. This study included six patients with Child's class $B$ cirrhosis and one patient with pre-sinusoidal portal hypertension for which the effects on esophageal motor function were evaluated both pre- and post-EVL. ${ }^{17}$ Whereas band ligation appears to have little impact on esophageal motility data are limited and hampered by lack of standardization rendering conclusions about safety premature. ${ }^{18}$ Larger studies that evaluate patients for esophageal motility disorders at least 3-6 months after EVL are needed.

There are some limitations to our study. While we identified 68 people who completed EVL, a number were lost to follow up after treatment ultimately decreasing our sample size. Second, our study only looks at EVL morbidity without an EVS arm. However, a prospective, randomized trial comparing EVL with EVS to evaluate long-term complications such as dysphagia would be difficult to perform given that most institutions no longer perform EVS routinely. Third, this was a single center study with a relatively small number of patients. Involving multiple institutions may allow for a more broad application of our findings. In addition, we incorporated a modified version of the MDQ that has not been previously validated. We chose this method of categorizing symptoms because no other suitable questionnaire for our purposes existed. Other studies have had this similar limitation such as studies published by Viazis et al. ${ }^{4}$ and Goff et al. ${ }^{8}$, where both studies utilized ad hoc dysphagia questionnaires.

In summary, dysphagia appears to be a common, long-term complaint a physician may encounter post-EVL. We were unable to identify any independent predictors of post-EVL dysphagia in regression analysis which controlled for several relevant potential confounders. Our study is important as only short-term esophageal symptoms after EVL have been previously reported. The mechanism resulting in dysphagia needs to be further clarified in larger, long-term studies.

\section{Authors' contribution}

Saraswathi Arasu: study concept and design; data entry; analysis and interpretation of data; drafting of manuscript

Hammad Liaquat: data entry; analysis and interpretation of data Jaspreet Suri: study concept and design; data entry; analysis and interpretation of data; drafting of manuscript

Adam Ehrlich: study concept and design; analysis and interpretation of data; critical revision of the manuscript for important intellectual content; study supervision

Frank Friedenberg: study concept and design; analysis and interpretation of data; critical revision of the manuscript for important intellectual content; study supervision

\section{Conflicts of Interest}

The authors have no conflicts to disclose.

\section{REFERENCES}

1. Garcia-Tsao G, Sanyal AJ, Grace ND, Carey W; Practice Guidelines Committee of the American Association for the Study of Liver Diseases; Practice Parameters Committee of the American College of Gastroenterology. Prevention and management of gastroesophageal varices and variceal hemorrhage in cirrhosis. Hepatology 2007;46:922-938.

2. Laine L, Cook D. Endoscopic ligation compared with sclerotherapy for treatment of esophageal variceal bleeding. Ann Intern Med 1995;123:280-287

3. Saeed ZA, Michaletz PA, Winchester CB, Woods KL, Dixon WB, Hieser $M C$, et al. Endoscopic variceal ligation in patients who have failed endoscopic sclerotherapy. Gastrointest Endos 1990;36:572574.

4. Viazis N, Armonis A, Vlachogiannakos J, Rekoumis G, Stefanidis G, Papadimitriou $\mathrm{N}$, et al. Effects of endoscopic variceal treatment on oesophageal function: a prospective, randomized study. Eur J Gastroenterol Hepatol 2002;14:263-269.

5. Sorensen T, Burcharth F, Pedersen ML, Findahl F. Oesophageal stricture and dysphagia after endoscopic sclerotherapy for bleeding varices. Gut 1984:25:473-477.

6. Sauerbruch T, Wirsching R, Leisner B, Weinzierl M, Pfahler M, Paumgartner $\mathrm{G}$. Esophageal function after sclerotherapy of bleeding varices. Scand J Gastroenterol 1982;17:745-751.

7. Ogle SJ, Kirk CJ, Bailey RJ, Johnson AG, Williams R, Murray-Lyon $I M$. Oesophageal function in cirrhotic patients undergoing injection 
sclerotherapy for oesophageal varices. Digestion 1978;18:178-185.

8. Goff JS, Reveille RM, Van Stiegmann G. Endoscopic sclerotherapy versus endoscopic variceal ligation: esophageal symptoms, complications and motility. Am J Gastroenterol 1988;83:1240-1244.

9. Van Stiegmann G, Goff JS. Endoscopic esophageal varix ligation: preliminary clinical experience. Gastrointest Endosc 1988;34:113117.

10. Shaheen NJ, Stuart E, Schmitz SM, Mitchell KL, Fried MW, Zacks $\mathrm{S}$, et al. Pantoprazole reduces the size of postbanding ulcers after variceal band ligation: a randomized controlled trial. Hepatology 2005:41:588-594.

11. McElhiney J, Lohse MR, Arora AS, Peloquin JM, Geno DM, Kuntz MM, et al. The Mayo Dysphagia Questionnaire-30: documentation of reliability and validity of a tool for interventional trials in adults with esophageal disease. Dysphagia 2010;25:221-230.

12. Schuman BM, Beckman JW, Tedesco FJ, Griffin JW Jr, Assad RT. Complications of endoscopic injection sclerotherapy: a review. Am J Gastroenterol 1987;82:823-830.
13. de Franchis R, Primignani M. Endoscopic treatments for portal hypertension. Semin Liver Dis 1999;19:439-455.

14. Villanueva C, Colomo A, Aracil C, Guarner C. Current endoscopic therapy of variceal bleeding. Best Pract Res Clin Gastroenterol 2008;22:261-278.

15. Van Stiegmann G, Goff JS, Sun JH, Hruza D, Reveille RM. Endoscopic ligation of esophageal varices. Am J Surg 1990;159:21-25; discussion 25-26.

16. Poza Cordon J, Froilan Torres C, Burgos García A, Gea Rodriguez F, Suárez de Parga JM. Endoscopic management of esophageal varices. World J Gastrointest Endosc 2012;4:312-322.

17. Berner JS, Gaing AA, Sharma R, Almenoff PL, Muhlfelder T, Korsten MA. Sequelae after variceal ligation and sclerotherapy: a prospective randomized study. Am J Gastroenterol 1994;89:852-858.

18. Fass R, Landau O, Kovacs TO, Ippoliti AF. Esophageal motility abnormalities in cirrhotic patients before and after endoscopic variceal treatment. Am J Gastroenterol 1997;92:941-946. 